\title{
Endonasal endoscopic approach for removal of intranasal nasal glial heterotopias*
}

\author{
N.-X. Bonne', S. Zago², G. Hosana', M. Vinchon³, T. Van Den Abbeele², and \\ P. Fayoux
}

Department of Pediatric Otolaryngology, Jeanne de Flandre Children Hospital, University Hospital of Lille, Lille, France

Department of Pediatric Otolaryngology, Robert Debré Children Hospital, University Hospital, Paris, France

Department of Pediatric Neurosurgery, Roger Salengro Hospital, University Hospital of Lille, Lille, France
Rhinology 50: 211-217, 2012

DOI:10.4193/Rhino11.108

* Received for publication:

May 29, 2011

Accepted: February 1, 2012

\section{Summary}

Background: Nasal Glial Heterotopias also called Nasal Gliomas (NG) are rare congenital tumours of the midline frontonasal space arising from a normal neurectodermal tissue entrapped during the closure of the anterior neuropore. Historically, such tumours were approached using a frontal craniotomy. The study aims to evaluate a fully endonasal endoscopic approach for intranasal NG removal.

Methods: We report a retrospective study of intranasal and mixed NG treated using endonasal endoscopic techniques and computer assisted navigation system from 1997 to 2010 in two tertiary referral centres of Paediatric Otolaryngology. All tumours were investigated using two imaging modalities: craniofacial MRI and CT-scan.

Results: Fifteen patients were included (0 to 14 years of age). All tumours were totally removed and no recurrence was observed after a mean follow-up of 32 months. A skull base plasty was done in 13 cases to cover a bony defect or to treat a cerebrospinal leak. Nasal packing was usually removed 24 hours after surgery and all children were discharged home after 2 to 4 days.

Conclusion: Removal of intranasal NGs using an endonasal endoscopic approach and a dedicated computer assisted navigation system is a safe and efficient procedure. Early management is recommended to treat neonatal airway obstruction.

Key words: nasal glioma, nasal obstruction, natural orifice endoscopic surgery, computer-assisted surgery, infant

\section{Introduction}

Midline congenital fronto-nasal masses include a heterogeneous subset of pathologies, in which anterior neuropore abnormalities, such as nasal glioma, encephalocele and dermoids, can be individualized from the other entities, including infantile haemangioma, lipoblastoma, and teratoid polyp.

From an embryological standpoint, the anterior neuropore appears between the two optic recesses during the third day of foetal life. Postnatal maturation of the anterior skull base requires a complex developmental process requiring adequate neuropore closure. During the first eight weeks of gestation, the proper closure of the anterior neuropore creates three transient anatomic landmarks: the fonticilus frontalis (a fontanel between the frontal bone and the nasal bones), the foramen cecum (a midline opening anterior to the crista galli of the ethmoid bones) and the prenasal space ${ }^{(1)}$. The prenasal space encloses a dural diverticulation lying above and anterior to the nasal septum, below and posterior to the nasal and frontal bones. Occasionally, this dural diverticulation becomes entrapped during the closure of the anterior neuropore resulting in the formation of either an encephalocele or a Nasal Glioma (NG). NG might link the intracranial dura mater through the foramen cecum and evidence of this connection is 
observed in about $15 \%$ of histopathological studies and referred as a 'fibrous stalk' (2).

From a histopathological standpoint, NG is defined by the combination of a dysplastic neuroglial tissue mixed with a fibrovascular tissue and an overlying epithelium ${ }^{(2)}$. Thus, many authors suggest calling these tumours Nasal Glial Heterotopias. Three clinical forms of NG are described based on their anatomic relationship with the nasal bones. The extranasal form (60\%) is superficially located, and expands into the subcutaneous space (3) Intranasal NG, the topic in this study, account for $30 \%$ of all NG seen at birth and are frequently revealed by respiratory distress in the early life. Mixed form (10\%) associates both extranasal and intranasal extensions ${ }^{(4)}$.

In the early management of NG, bifrontal craniotomy has been recommended ${ }^{(4,5)}$. Although, historically, a craniotomy was recommended to safely investigate any intracranial connection in the absence of accurate diagnostic test, since, there has been critical improvement in imaging, surgical planning and monitoring ${ }^{(6-9)}$, and a recent interest in improving and evaluating endoscopic endonasal surgery to treat skull base lesions and CSF leak in children ${ }^{(10-12)}$. These improvements in technology, surgical technique, and medical environment result in introducing a single staged endoscopic endonasal procedure in the treatment of NG. Such an approach has been published in some recent case reports or short series that include other pathologies ${ }^{(13-22)}$. Nevertheless, case reports lack either follow-up or accurate surgical records, and do not assess the reproducibility of a technique. Therefore, we investigated the result of this surgical technique in a case series focusing on safety and follow-up results.

\section{Materials \& methods}

\section{Study}

From 1997 to 2010, 19 patients were admitted in two tertiary referral centres of paediatric otolaryngology for NG. From these nineteen, fifteen underwent endoscopic endonasal removal of an intranasal or mixed NG. After IRB approval, medical records and imaging studies provided data concerning the clinical presentation, surgical technique and complication. This study aims to evaluate endonasal endoscopic approach for removal of intranasal NG followed with skull base plasty.

\section{Surgical procedure and post-operative care}

All the procedures were conducted using $2.7 \mathrm{~mm}, 30^{\circ}$ endoscopes introduced transnasally. In our experience, we do not recommend the systematic use of an irrigation sheath because it limits the accessibility to the anterior ethmoidal roof due to the nostril diameter in newborn children before the age of $3^{(21)}$. For older children, we used a combination of $30^{\circ}$ and $70^{\circ}, 4.0 \mathrm{~mm}$ endoscopes.
Under general anaesthesia, we first proceeded in a bilateral nasopharyngeal endoscopy to identify any potential cerebrospinal fluid (CSF) leak or abnormal mass pulsatility. This examination time was sensitized using Vasalva manoeuvre. When accessible, the ipsilateral middle turbinate was lateralized to visualize the cribriform plate behind the mass. In most of the surgeries, secondary to mass effect, the nasal septum was lateralized to the opposite side thus allowing a larger field than first expected. NG removal was then progressively achieved with tumour debulking and meticulous bipolar haemostasis to keep the operative field free of bleeding. During this time the overlying mucosal strip was removed 'en bloc' without attempt to separate from the tumour capsule. The piecemeal dissection was continued until the fibrous stalk could be reached. Further exposure included identification of the triangular cartilage of the nasal septum in the midline, the cribiform plate posteriorly, the nasal bones laterally and the foramen cecum anteriorly. Thereby, it allowed the section of the stalk just below the anterior skull base dura (Figure 1). For smaller skull base defects, the head of either the middle or inferior turbinate was harvested and filleted to expose its bony area and this was used as an onlay graft. Alternatively, we tailored neurovascular pedicle flaps based on the anterior ethmoidal artery. In cases exhibiting high flow CSF leaks, prior to interposing the flap, a thick conchal chondro-perichondral graft was used for defect repair in our series. Grafts were then secured with fibrin glue and stabilized with a Silastic roll. Children were allowed to recover from anaesthesia and observed 2 hours in a specialized Paediatric Intensive Care Unit (PICU) before being discharged to the department of paediatric otolaryngology.

\section{Computer-assisted navigation system}

After 2000 in one centre and 2004 in the other, we used the commercially available system DIGIPOINTEUR (Collin-ORL, Bagneux, France), a low field electromagnetic localization device (Figure 2). Developed for endonasal surgery and neurotological surgery, its expected accuracy is in the sub-millimetre range (manufacturer information). After positioning the frontal headset, the skin surface is matched with the pre-operative CT-scan using a non-invasive handset and multiple plot records without dedicated fiducials. During surgery the handset is adapted to any instrument (i.e. suction) and allows continuous localization.

\section{Results}

\section{Patients}

From the nineteen patients referred for NG between 1997 and 2010, four cases of extra-nasal NGs operated on using a rhinoplasty approach were excluded, thirteen patients presenting with an intranasal NG, and two cases of mixed NG were included in our study. No sex prevalence was observed (8:7) and no associated malformation was reported. In all cases, physical examination revealed a greyish nasal polyp and anterior discharge. Three tumours were diagnosed at birth. Eight NG were 


\section{A}

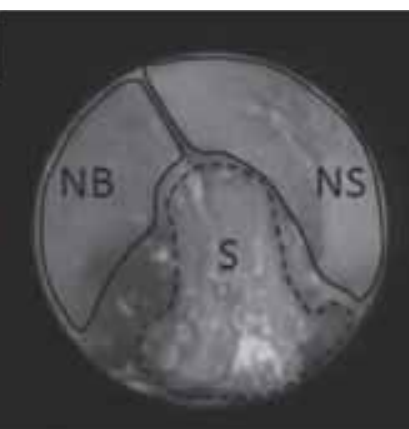

B

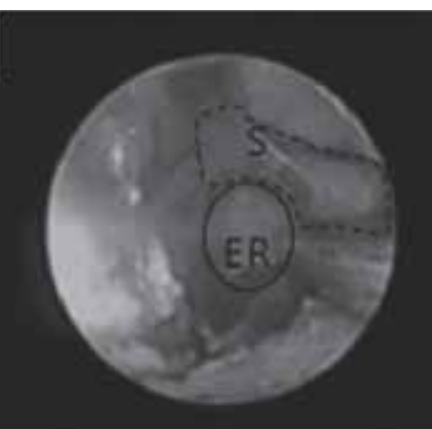

c

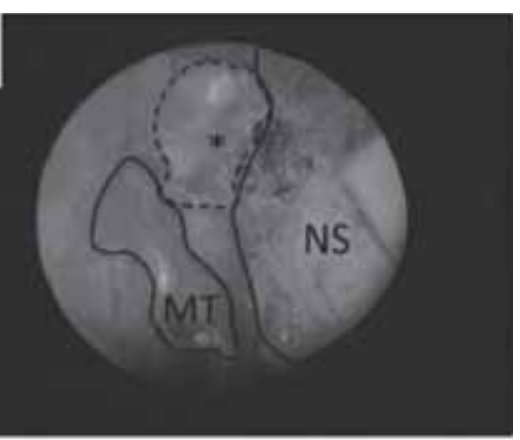

Figure 1. Endoscopic view after tumour debulking, right nasal fossa. [A] Implantation of the stalk (S, dashed line) is identified and separated from the nasal septum (NS) and right nasal bone (NB). [B] The ethmoidal roof is then identified (ER). [C] Endocopic view after skull base plasty using a mucoosteoperiostal free graft $\left({ }^{*}\right.$, dashed line) harvested from the middle turbinate (MT).
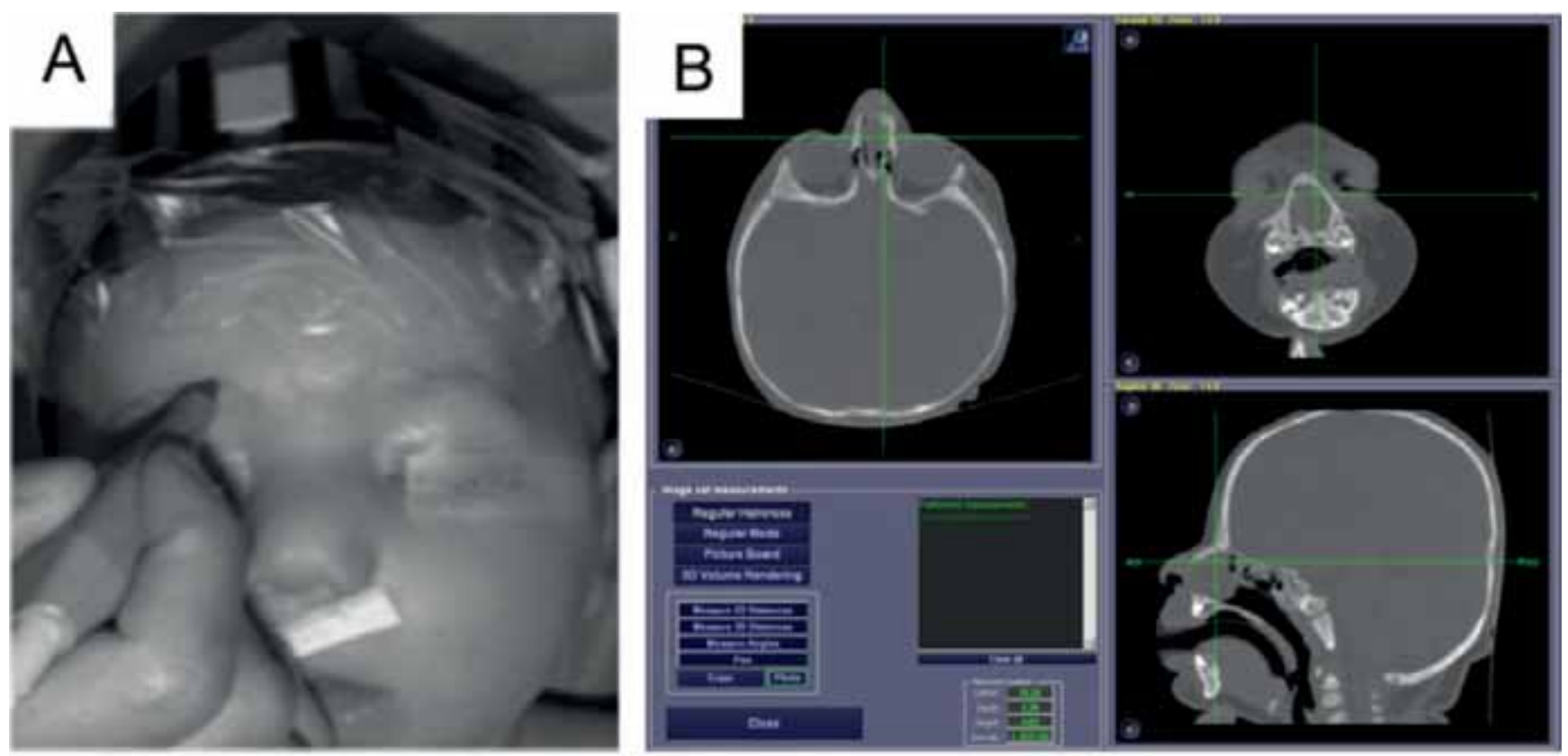

Figure 2. [A] Surface registration is performed after positioning the frontal headset. [B] Regular haircross mode obtained during navigation for removal of a left nasal glioma. Location of the stalk is obtained. Note in this example that the central skull base is almost entirely ossified.

revealed by nasal obstruction (53\%) that was either isolated and well tolerated (2/8), resulted in snoring (3/8), or caused acute upper-airway obstruction associated dyspnoea (3/8). Episodes of dyspnoea that were noted in these three patients occurred at birth in one, at 8 days of life (with associated feeding difficulties) in another, and at 10 months (with associated cyanosis) in the third. One patient presented with chronic rhinosinusitis. One tumour remained asymptomatic for 14 years of life.

All tumours but one (14/15) were removed by the age of 18 months. For those 14 cases, the mean age at the time of surgery was 8.9 months. The delay applied after diagnosis until surgery averaged 6.4 months ( 0.1 to 18 months). Because of solely nasal breathing in the newborn child, NG should be considered as a possible diagnosis in infants presenting with dyspnoea. Dyspnoea occurs secondary to tumour growth on one side and mass effect on the septum on the other side of the nasal airway. Consequently, respiratory tolerance guided the extent of observation time before surgery. Occurrence of dyspnoea secondary to upper airway obstruction motivated accelerated surgical treatment in 3 patients to restore patent nasal fossa with no limitation of age (i.e., the youngest patient in the series was 2 days old). 


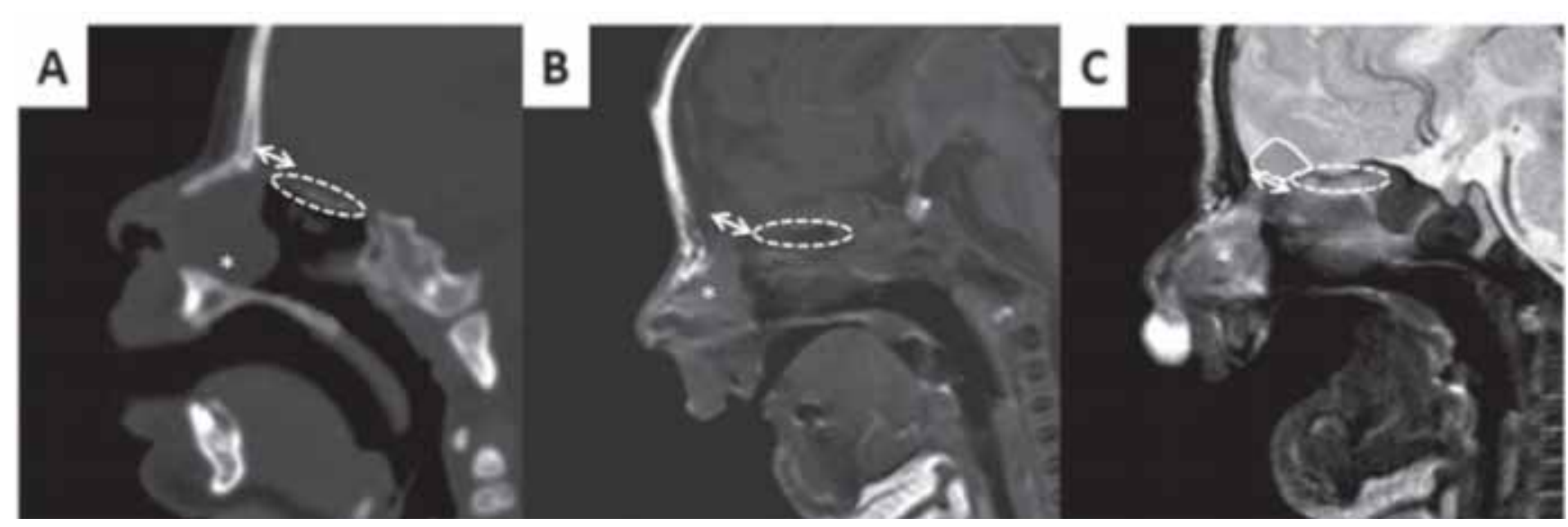

Figure 3. Preoperative imaging. [A] CT-scan: the anterior skull base is partially ossified (dashed ellipse) except at the foramen cecum (arrow). [B, C] MRI ( $\mathrm{T} 1$ and $\mathrm{T} 2$ weighted images) of the same case highlights the relationship between the nasal glioma ${ }^{*}$ ) and the anterior skull base (dashed ellipse). Although no connection to the sub-arachnoidal space was described, a stalk is identified connecting the tumour to the foramen cecum (arrow) anterior to the apophyse crista galli (delineated).

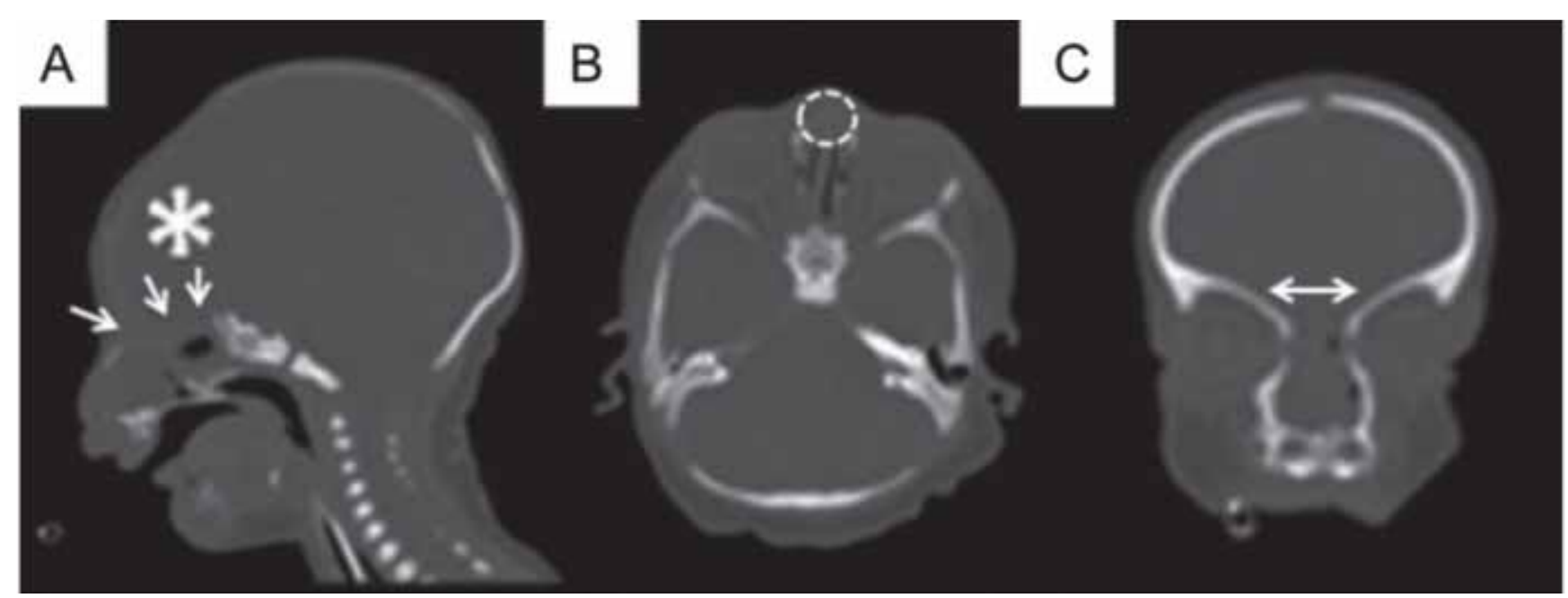

Figure 4. Screenshot obtained during navigation after multiplanar reconstruction of a 3 days old child's CT-scan. [A] Note the absence of ossification in the midline $\left(^{*}\right)$ and of the anterior skull base (arrows). [B] Anterior location of the tumour (dashed line). [C] There was a total absence of ossification in the area of the cribriform plate and ethmoid bone (double arrow).

\section{Surgery and follow-up}

All patients benefited from the described endonasal endoscopic technique for removal of their intranasal NG. During surgery, the bleeding was easily controlled using bipolar cautery. A fibrous stalk connecting the tumour to the dura was identified in $40 \%$ of the cases $(6 / 15)$, CSF leak was noted perioperatively in $66.6 \%$ of these cases (4/6). After skull base plasty, recurrence of the leak was noted the day after in one case and motivated revision surgery for adjunction of an inlay cartilage graft. One child experienced post-operative dyspnoea and was observed 24 hours in the PICU before being discharged to the department of paediatric otolaryngology. For 7 (47\%) patients treated by the same team (TVA), the nasal packing was maintained 2 days in 2 patients and 3 days in 4, whereas it was maintained only 24 hours for 8 children (53\%) treated in the other institution (PF) without impairing the outcomes (i.e., no secondary CSF leak in this group).

During the long-term follow-up period (mean of 32 months), neither tumour nor CSF leak recurred. However, the patient who originally presented with a CSF recurrence developed pneumococcal meningitis 5 years after surgery. Physical examination 
before and at the time of meningitis did not reveal any discharge and confirmed a complete healing of the mucosal flap. Cysterno-MRI did not reveal any nasal hypersignal on T2 weighted images. Following these investigations, we determined that a late recurrence of CSF leak was unlikely although this hypothesis was considered.

\section{Imaging}

MRI was the modality of choice used in this series to explore the relationship between the tumour and brain parenchyma (Figure 3). In all cases, MRI revealed the glial heterotopias as a mass with iso-intense signal in $\mathrm{T} 1$ and marked hyperintense $\mathrm{T} 2$ signal. Use of contrast medium showed uptake at the periphery of the lesion consistent with a mucosal wall. In one case, however, the tumour was thought to be a meningocele rather than a NG. Computed tomography comprised the initial assessment and focused on investigating skull base ossification (Figure 4). The standard procedure was a spiral acquisition without infusion of contrast medium. This acquisition was dedicated for use with the computer navigation system as well.

\section{Pathology}

Pathological findings were consistent with nasal glial heterotopia in all cases showing association of a vascular stroma tissue with neuroglial cells. However, in two tumours, distinction from an encephalocele remains inconclusive due to mixing of areas consistent with nasal glial heterotopia and others revealing the presence of neurons. Rahbar et al., discussed this pathological finding, emphasizing difficulties in diagnosis, and concluded that gliomas should be distinguished from encephalocele only after clinicopathological correlation ${ }^{(15)}$.

\section{Discussion}

In 1963, Walker and Resler reviewed the surgical treatment of NG and recommended a two-staged procedure ${ }^{(4)}$. The first stage would focus on accessing the anterior skull base through a bifrontal craniotomy to exclude a fibrous stalk or treat a misdiagnosed encephalocele. Moreover, this approach was believed beneficial to accurately reinforce the skull base in case of osteodural defect. The removal of the nasal mass would be delayed to a second stage via a transfacial route, providing an intact skull base. According to these authors, the cosmetic result of a bifrontal craniotomy for intranasal NG removal was satisfying. Therefore, access through this approach was recommended as a diagnostic test and therapeutic option ${ }^{(4,5)}$. However, approaching the anterior skull base through a bifrontal craniotomy requires some extent of frontal lobe retraction exposing to the risk of frontal lobe oedema or hematoma ${ }^{(22)}$. Moreover, the exposure might be inadequate, as illustrated recently by Wu et al., who reported the case of a 9 day-old child for whom endonasal removal of a residual intranasal NG was achieved after failure of a prior attempt through the transcranial approach ${ }^{(13)}$. Altogether, considering the risks of cranial surgery, the subsequent need for endonasal removal in some patients, and the development of minimally invasive surgery resulted in proposing alternative approaches. Transfacial approaches, although introducing further cosmetic issues, were generally preferred to purely endonasal approaches because exposure was considered to be too narrow and to potentially increase the risk of meningitis in the latter. In fact, the rate of infection following endonasal endoscopic skull base surgical procedures has been recently assessed at $1.8 \%$, in a large number of patients and is comparable to that observed in open craniotomy ${ }^{(23)}$. In particular, this series highlights that postoperative CSF leak is the most important risk factor, as was observed in one of our patients.

\section{Endoscopic endonasal approach}

During the past ten years, many publications reported the use of endoscopic endonasal surgery for NG removal. Unfortunately, some are reported in national language and, therefore, were excluded from our analysis. To our knowledge, Rahbar et al. reported the only series including two cases of intranasal NG ${ }^{(15)}$. Although they did not report any complication or recurrence, the follow-up was only 3 months in one patient. In five published case reports with more than 2 years of follow-up, no complication or recurrence was noted ${ }^{(13,15,16,19,21)}$. The use of combined imaging (MRI and CT-scan) has allowed a better selection of patients eligible for endoscopic surgery and was equally used in those reports ${ }^{(13-19,21,22)}$. In the reviewed articles published in the past ten years, a fibrous stalk was noted in 3/11 (27\%) suggesting a higher rate of connection between the tumour and the dura than expected. This difference is consistent with our series and might reflect a selection bias of paediatric otolaryngology centres.

\section{Imaging}

On T1-weighted images, NG are hypointense to isointense to gray matter. On T2-Weighted images, T2 hyperintensity caused by gliosis is often observed within the mass. Dysplastic tissue does not typically enhance. Any connection to the anterior cranial fossa can be assessed, and it enables simultaneous assessment of associated brain anomalies. Diffusion imaging sequence is usually added to differentiate NG from epidermoid cysts ${ }^{(6)}$. Using CT-scan, NG appears as nonspecific soft tissue masses that do not enhance after contrast administration. However, nasal processes of the frontal bones, nasal bones and the ethmoidal roof are not ossified at birth. The ossification of the anterior skull base will proceed rapidly during the first 6 months and the crista galli will start its ossification as early as 2 months of age. However, an anterior gap located between the ethmoid and the nasal bones will persist. Although $84 \%$ of the anterior skull base will be ossified by 2 years, only 4 - $8 \%$ of the children will have a complete ossification of the anterior margin of the cribriform plate at this time ${ }^{(25,26)}$. The full ossification of the anterior skull 
base could is not completed before 4 years of age ${ }^{(25-27)}$. Thereby CT-scan is not felt as a reliable tool to investigate the connection between NG and the intracranial compartment as reported previously for nasal dermoids ${ }^{(28)}$. Finally, this delayed ossification of the skull base may result in overestimating the size of the bony defect on CT-scan examination (11).

\section{Skull base plasty}

The choice of the graft is guided by three main elements: age at surgery, size of the bony defect and magnitude of the leak. We believe that neurovascular pedicle mucoperichondrium flaps are more likely to engraft successfully. However, Shah et al., based on anatomical studies of the paediatric skull base, reported that a neurovascular-pedicle flap, based on the nasoseptal artery, should not be used to reconstruct anterior skull base in children prior to nine years of age ${ }^{(29)}$. Nevertheless, the vasculature of the septal mucosa allows tailoring pedicle flaps to cover the defects of the skull base seen in NG removal. As previously reported, the use of inlay conchal chondro-perichondral grafts increases the stability and seal of the cranioplasty, preventing CSF leak ${ }^{(11)}$.

\section{Computer assisted navigation system}

Few reports evaluate the precision of a navigation system in paediatric skull base surgery ${ }^{(7-9)}$. In this report, and in contrast with systems previously published, pre-operative CT-scans are obtained without wearing the headset. Indeed, most of the patients are referred with a previous CT-scan that can be used if compatible with the navigation system. Other system may require a new acquisition with subsequent increased exposure to $X$-ray irradiation. During surgery, the handset is adapted on any instrument, including the suction. Therefore, it allows anatomical mapping simultaneously with surgery.

The series reported herein assesses the benefit of endoscopic surgery in the management of intranasal NG. As neither acute complication nor long term recurrence is reported, we do recommend the use of endonasal endoscopic surgery for intranasal NG removal. Meanwhile, according to our series, the paediatric airway should be carefully monitored during recovery, particularly in younger patients. Finally, we recommend a long-term follow up with yearly examination.

\section{Author Contribution}

Study concept and design: N-XB, PF; Acquisition of data: N-XB, $\mathrm{SZ}, \mathrm{GH}$; Analysis and interpretation of data: N-XB, SZ, GH; Drafting of the manuscript: N-XB, TvdA, PF; Critical revision of the manuscript for important intellectual content: MV; Study supervision: TvdA, PF

\section{Conflict of interest statement}

No conflict of interest to report.

\section{References}

1. Charrier JB, Leboulanger N, Roger G, Denoyelle F, Garabédian EN, Monteil JP. Nasal glial heterotopia: embryological and clinical approaches. Rev Stomatol Chir Maxillofac. 2006; 107: 44-49.

2. Patterson K, Kapur S, Chandra RS. Nasal gliomas and related brain heterotopias: a pathologist's perspective. Pediatr Pathol. 1986; 5: 353-362.

3. Thomson HG, al-Qattan MM, Becker LE. Nasal glioma: is dermis involvement significant? Ann Plast Surg. 1995; 34: 168-172.

4. Walker EA jr, Resler DR. Nasal glioma. Laryngoscope. 1963; 73: 93-107.

5. Hughes GB, Sharpino G, Hunt W, Tucker $\mathrm{HM}$. Management of the congenital midline nasal mass: a review. Head Neck Surg. 1980; 2: 222-233.

6. Hedlund G. Congenital frontonasal masses: developmental anatomy, malformations, and MR imaging. Pediatr Radiol. 2006; 36: 647-662.

7. Klimek L, M Vosges R, Laborde G, Korves B. Computer-assisted image-guided surgery in pediatric skull-base procedures. J Pediatr Surg. 1995; 30: 1673-1676.

8. Postec F, Bossard D, Disant F, Froehlich P. Computer-assisted navigation system in pediatric intranasal surgery. Arch Otolaryngol Head Neck Surg. 2002; 128: 797-800.
9. Parikh SR, Cuellar H, Sadoughi B, Aroniadis $\mathrm{O}$, Fried MP. Indications for image-guidance in pediatric sinonasal surgery. Int J Pediatr Otorhinolaryngol. 2009; 73: 351-356.

10. Kassam A, Thomas AJ, Snyderman C, et al. Fully endoscopic expanded endonasal approach treating skull base lesions in pediatric patients. J Neurosurg. 2007; 106 (2Suppl): 75-86.

11. Van Den Abbeele T, Elmaleh M, Herman $P$, François M, Narcy P. Transnasal endoscopic repair of congenital defects of the skull base in children. Arch Otolaryngol Head Neck Surg. 1999; 125: 580-584.

12. Locatelli D, Rampa F, Acchiardi I, Bignami M, Pistochini A, Castelnuovo P. Endoscopic endonasal approaches to anterior skull base defects in pediatric patients. Childs Nerv Syst. 2006; 22: 1411-1418.

13. Wu CL, Tsao LY, Yang AD, Chen MK. Endoscopic surgery for nasal glioma mimicking encephalocele in infancy. Skull Base. 2008; 18: 401-404.

14. Sciarretta $V$, Pasquini $E$, Frank $G$, et al. Endoscopic treatment of benign tumors of the nose and paranasal sinuses: a report of 33 cases. Am J Rhinol. 2006; 20: 64-71.

15. Rahbar R, Resto VA, Robson CD, et al. Nasal glioma and encephalocele: diagnosis and management. Laryngoscope. 2003; 113: 2069-2077.

16. Yokoi H, Wada R, Ichikawa G. Endoscopic treatment of so-called intranasal glioma. Rhinology. 2002; 40: 217-219.

17. Rouev P, Dimov P, Shomov G. A case of nasal glioma in a new-born infant. Int J Pediatr Otorhinolaryngol. 2006; 58: 91-94.

18. Dimov P, Rouev P, Tenev K, Krosneva R, Valkanov P. Endoscopic surgery for the removal of a nasal glioma: case report. Otolaryngol Head Neck Surg. 2001; 124: 690.

19. Yokoyama M, Inouye N, Mizuno F. Endoscopic management of nasal glioma in infancy. Int J Pediatr Otorhinolaryngol. 1999; 51:51-54.

20. Burckhardt W, Tobon D. Endoscopic approach to nasal glioma. Otolaryngol Head Neck Surg. 1999; 120: 747-748.

21. Pasquini E, Farneti G, Giausa G, Biavati M. A rare case of nasal glioma in adult age. Otolaryngol Head Neck Surg. 1998; 118: 905-906.

22. Greenfield JP, Anand VK, Kacker A, et al. Endoscopic endonasal transethmoidal transcribriform transfovea ethmoidalis approach to the anterior cranial fossa and skull base. Neurosurgery. 2010; 66: 883-892.

23. Raza SM, Conway JE, Li KW, et al. A modified frontal-nasal-orbital approach to midline lesions of the anterior cranial fossa and skull base: technical note with case illustrations. Neurosurg Rev. 2010; 33: 63-70.

24. Kono Y, Prevedello DM, Snyderman $\mathrm{CH}$, et al. One thousand endoscopic skull base 
surgical procedures demystifying the infection potential: incidence and description of postoperative meningitis and brain abscesses. Infect Control Hosp Epidemiol. 2011; 32: 77-83.

25. Belden CJ, Mancuso AA, Kotzur IM. The developing anterior skull base: $\mathrm{CT}$ appearance from birth to 2 years of age. AJNR Am J Neuroradiol. 1997; 18: 811-818.

26. Hughes DC, Kaduthodil MJ, Connolly DJ, Griffiths PD. Dimensions and ossification of the normal anterior cranial fossa in children. AJNR Am J Neuroradiol. 2010; 31: 1268-1272.

27. Tatreau JR, Patel MR, Shah RN, et al. Anatomical considerations for endoscopic endonasal skull base surgery in pediatric patients. Laryngoscope. 2010; 120: 1730-1737.
28. Pensler JM, Bauer BS, Naidich TP. Craniofacial dermoids. Plast Reconstr Surg. 1988; 82: 953-958.

29. Shah RN, Surowitz JB, Patel MR, et al. Endoscopic pedicled nasoseptal flap reconstruction for pediatric skull base defects. Laryngoscope. 2009; 119 (6): 1067-1075.

\section{Dr Pierre Fayoux \\ Department of Pediatric}

Otolaryngology

Hôpital Jeanne de Flandre

CHRU Lille - Avenue Eugène Avinée

59037 Lille Cedex

France

Tel: +33-(0)3-2044 5067

Fax: +33-(0)3-2044 4656

Email: pierre.fayoux@chru-lille.fr

\section{ADVERTISEMENT}

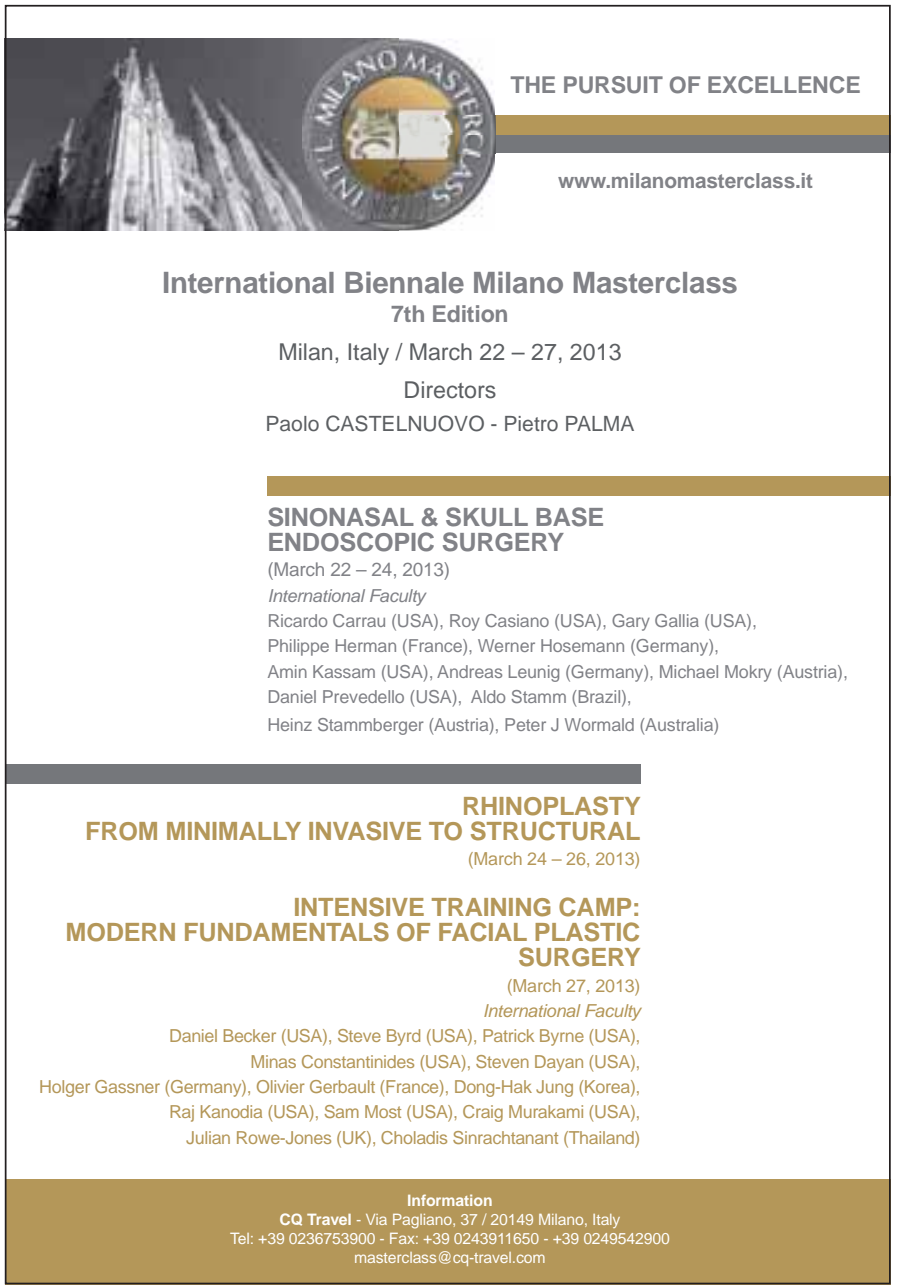

